\title{
THE STANDARD SUMMATION OPERATOR, THE EULER-MACLAURIN SUM FORMULA, AND THE LAPLACE TRANSFORMATION
}

\author{
JOHN BORIS MILLER
}

(Received 13 April 1984)

Communicated by J. H. Chabrowski

\begin{abstract}
A proof is given of the Euler-Maclaurin sum formula, on a Banach space of differentiable vectorvalued functions of bounded exponential growth, using the Laplace transformation. Some related summation formulae are proved by the same methods. Properties of the standard summation operator are proved, namely spectral properties and boundedness, continuity and differentiability results.

1980 Mathematics subject classification (Amer. Math. Soc.): primary 65 B 15, 44 A 10, 47 B 38; secondary 47 B 38, 39 B 70.

Keywords and phrases: standard summation operator, Euler-Maclaurin formula, Laplace transformation, functions of exponential growth, differentiation operator, integration operator, Bernoulli polynomials, periodic extension, periodic Bernoulli polynomials, functional equation.
\end{abstract}

\section{Introduction}

The aim of this paper is to give a new proof of the classical Euler-Maclaurin formula using Laplace transform techniques, and to describe some properties of the standard summation operator on spaces of differentiable functions. Here we stretch the adjective 'classical' to include the case of vector-valued functions; but this is only an incidental generalization, and not the main purpose of the proof. The new proof of the Euler-Maclaurin formula is no shorter than existing proofs, but it does show very clearly the close connection between the formula and the Laplace transformation; it is based upon an elegant relation between the Laplace transforms of the $n$th Bernoulli polynomial and its periodic extension (Lemma 4).

(C) 1985 Australian Mathematical Society $0263-6115 / 85 \$ A 2.00+0.00$ 
The proof is a reformulation, for a space of differentiable functions, of a proof given previously by the author for generalized functions, in [8].

The formula is seen as a statement about the standard summation operator $S_{\omega}$, defined by

$$
\left(S_{\omega} x\right)(t)=\sum_{r=0}^{[t / \omega]} x(t-r \omega) ;
$$

indeed it can be thought of as a Laurent series expansion for this operator in terms of a variable $\omega$ about 0 , although we allow only $\omega>0$. In an earlier paper [7] (see also [5], [6], [9]) the author discussed the Euler-Maclaurin formula arising from a general Baxter operator acting on an arbitrary Banach algebra. Because of the spectral conditions required there, the operator $S_{\omega}$ in (1), although a Baxter operator, is not covered by that discussion, and the present paper is in part an attempt to clarify this inadequacy. The choice of Banach space on which to study $S_{\omega}$ is suggested by the Euler-Maclaurin formula: it is $\mathbb{E}_{0}^{n}\left(\mathbb{R}^{+}, X ; \rho\right)$, the space of $n$ times continuously differentiable functions of exponentially bounded growth taking values in a Banach space $X$, and vanishing with their derivatives at 0 . On this $\mathbb{E}_{0}^{n}, S_{\omega}$ turns out rather surprisingly to be bounded, and something can be said about its spectrum and about the properties of the function $\omega \mapsto S_{\omega}$. We give a formula for the resolvent of $S_{\omega}$. The spectrum does not contain 0 , and $S_{\omega}^{-1}$ is a difference operator (Theorem 5).

The properties of the function $\omega \mapsto S_{\omega}$ depend upon a functional equation (Lemma 8) which it satisfies. Continuity between spaces is uniform, but on a single space the matter is more delicate. One is also interested in the existence of the limit $K=\lim _{\omega \backslash 0} \omega S_{\omega}$. The results are summarized in Theorem 9.

Finally, in Section 6 some related summation formulae are proved by the same Laplace methods. These include Boole's summation formula and the periodic formulae of Berndt and Schoenfeld.

The author would like to thank for its hospitality the Faculty of Mathematical Studies at the University of Southampton, where this work was done.

\section{Notation and preliminary lemmas}

2.1 Let $X$ denote a complex Banach space, with norm $\|\cdot\|$. We use $X$-valued functions $x, y, \ldots$ on $\mathbf{R}^{+}=[0, \infty)$. The derivative $x^{\prime}(t)$ of $x$ is the limit in norm of $(x(t+h)-x(t)) h^{-1}$ as $h \rightarrow 0$ (or as $h \searrow 0$ when $t=0$; and one-sided derivatives are also used elsewhere) if this limit exists. Integrals of $X$-valued functions may be taken to be Bochner integrals (see [4], pp. 78ff); most integrands 
will be continuous functions. The Laplace transform of $x$ is the function $\hat{x}$ defined by

$$
\hat{x}(s)=\int_{0}^{\infty} e^{-s t} x(t) d t=\lim _{T \rightarrow \infty} \int_{0}^{T} e^{-s t} x(t) d t,
$$

whose domain includes some open halfplane of $\mathbf{C}$ and whose range is in $X$ ([4], Chapter 6, and [10]). The same formula (2) is used also for the Laplace transform $\hat{f}$ of a $\mathbf{C}$-valued function $f$.

First we summarize some well-known properties of Laplace transforms needed here.

Lemma 1. Let $x: \mathbb{R}^{+} \rightarrow X$ have continuous derivatives $x^{\prime}, x^{\prime \prime}, \ldots, x^{(k-1)}$ on $\mathbb{R}^{+}$, with $x^{(k-1)}$ absolutely continuous, satisfying

$$
\left\|x^{(j)}(t)\right\| \leqslant c e^{\rho t} \quad\left(\text { all } t \in \mathbb{R}^{+}\right)
$$

for some positive constants $c$ and $\rho$, and $j=0,1, \ldots, k-1$. Then the Laplace integrals $\left(x^{(j)}\right)^{\wedge}(s), j=0,1, \ldots, k$, converge (absolutely for $j \leqslant k-1$ ) for all $\operatorname{Re}(s)>\rho ;$ and for $j=1,2, \ldots, k$,

$$
\left(x^{(j)}\right)^{\wedge}(s)=-x(0) s^{j-1}-x^{\prime}(0) s^{j-2}-\cdots-x^{(j-1)}(0)+s^{j} \hat{x}(s) .
$$

If also $x(0)=x^{\prime}(0)=\cdots=x^{(k-1)}(0)=0$ then $s^{j} \hat{x}(s)$ is the (value at $s$ of the) Laplace transform of $x^{(j)}$ for $j=1,2, \ldots, k$ and $\operatorname{Re}(s)>\rho$.

Proof. By induction, using integration by parts.

LEMMA 2. If $x$ is continuous and the Laplace integral $\hat{x}(s)$ converges absolutely (i.e. if $\left.\int_{0}^{\infty} e^{-t \operatorname{Re}(s)}\|x(t)\| d t<\infty\right)$ then $s^{-1} \hat{x}(s)$ is the Laplace transform of $K x$, where

$$
(K x)(t)=\int_{0}^{t} x(u) d u
$$

LEMMA 3. If $x: \mathbb{R}^{+} \rightarrow X$ and $f: \mathbb{R}^{+} \rightarrow \mathbb{C}$ are continuous and the integrals $\hat{x}(s), \hat{f}(s)$ converge absolutely, then $\hat{f}(s) \hat{x}(s)$ is the Laplace transform of $\int_{0}^{t} f(t-u) x(u) d u$.

For the proofs of Lemmas 2 and 3 we can use [4], Theorem 6.2.4.

2.2 Definition. For $n \in \mathbb{N}$ and $\rho \in \mathbb{R}$ let $\mathbb{S}^{n}\left(\mathbb{R}^{+}, X ; \rho\right)$, or $\mathbb{C}^{n}$ for short, denote the space of all functions $x: \mathbb{R}^{+} \rightarrow X$ which have continuous derivatives up to and including $x^{(n)}$ on $\mathbb{R}^{+}$, satisfying (3) for $j=0,1, \ldots, n$. Here $c$ may depend upon $x$. 
Clearly $\mathbb{C}^{n}$ is a Banach space with respect to pointwise operations, and norm

$$
|x|_{n}=\sum_{j=0}^{n} \frac{1}{j !} \sup _{t \geqslant 0}\left(\left\|x^{(j)}(t)\right\| e^{-\rho t}\right)
$$

(note that the norm depends upon $\rho$ ).

$\mathbb{C}^{\infty}\left(\mathbb{R}^{+}, X ; \rho\right)$, or $\mathbb{C}^{\infty}$ for short, is the subset of $\bigcap_{n=1}^{\infty} \mathbb{E}^{n}\left(\mathbb{R}^{+}, X ; \rho\right)$ consisting of those $x$ for which $|x|_{\infty}$ is finite, and is a Banach space with respect to this norm.

$\mathbb{C}_{0}^{n}\left(\mathbb{R}^{+}, X ; \rho\right)$, briefly $\mathbb{E}_{0}^{n}$, for $1 \leqslant n \leqslant \infty$ denotes the closed subspace of $\mathbb{E}^{n}$ consisting of those $x$ for which $x^{(j)}(0)=0$ for $j=0,1,2, \ldots, n$. We write $[x]_{j}=$ $\sup _{t \geqslant 0}\left\|x^{(j)}(t) e^{-\rho t}\right\|$, so that

$$
\left\|x^{(j)}(t)\right\| \leqslant e^{p t}[x] ; \text { for all } t \geqslant 0,
$$

and $|x|_{n}=\sum_{j=0}^{n}[x]_{j} / j$ ! (The factorial factor is significant only when $n=\infty$ or when ring multiplication is under consideration.)

If $\rho \leqslant 0$ and $X$ is a Banach algebra then $\mho^{n}\left(\mathbb{R}^{+}, X ; \rho\right)$ is also a Banach algebra with respect to pointwise multiplication; if $\rho<0$ then ${ }^{n}{ }^{n}$ is without identity, and its elements are functions which together with their derivatives decay at least exponentially to zero as $t \rightarrow \infty$.

If $\rho>0$ then $\mathbb{S}^{n}$ is not an algebra. To obtain an algebra one would need to modify the definition, replacing $\rho$ by a sequence $\mathrm{P}=\left(\rho_{j}\right)_{j=0}^{\infty}$ of nonnegative numbers and writing $\rho_{j}$ instead of $\rho$ in the definition of $[x]_{j}$. Provided the sequence is superadditive, that is, $\rho_{j}+\rho_{k} \leqslant \rho_{j+k}$, and hence increasing and unbounded, ${ }^{n}$ so modified is a Banach algebra with identity which we denote by $\mathbb{E}^{n}(\mathrm{P})$. Even here we must have $\rho_{0}=0$, so that $x$ in $\mathbb{S}^{n}(\mathrm{P})$ is bounded though its derivatives may increase exponentially with $t$. If $\rho_{1}>0$ then the sequence $\mathrm{P}$ is strictly increasing. We do not use $\mathbb{C}^{n}(\mathrm{P})$ in this paper.

2.3 For the Bernoulli numbers and polynomials we follow the notation of [3, pp. 320, 321]. Thus the Bernoulli numbers are the constants $B_{j}$ in the formula

$$
\frac{e^{\lambda}}{e^{\lambda}-1}=\frac{1}{\lambda}+\frac{1}{2}+\sum_{j=1}^{\infty} \frac{(-1)^{j-1} B_{j} \lambda^{2 j-1}}{(2 j) !} \quad(0<|\lambda|<2 \pi)
$$

and the $n$th Bernoulli polynomial is

$$
\text { (9) } \begin{aligned}
\phi_{n}(t) & =t^{n}-\frac{1}{2} n t^{n-1}+\sum_{j=1}^{\left[\frac{1}{(}(n-1)\right]}(-1)^{j-1}\left(\begin{array}{c}
n \\
2 j
\end{array}\right) B_{j} t^{n-2 j} \quad(n=2,3, \ldots), \\
\phi_{1}(t) & =t .
\end{aligned}
$$

Here $[\cdot]$ is the greatest integer function, and empty sums are zero. Note that $\phi_{n}(0)=0$. The periodic extension of $\phi_{n}$ outside $[0,1)$ is written $\psi_{n}$ :

(10) $\psi_{n}(t)=\phi_{n}(t)$ for $0 \leqslant t<1, \quad \psi_{n}(t+r)=\psi_{n}(t)$ for $r=1,2, \ldots$ 
We shall use the recurrence formula

$$
\phi_{n}(t+1)-\phi_{n}(t)=n t^{n-1} \quad(n=1,2, \ldots) .
$$

LEMMA 4. The Laplace transform integrals of $\phi_{n}$ and $\psi_{n}$ converge absolutely for $\operatorname{Re}(s)>0$, and

$$
\hat{\phi}_{n}(s)=\hat{\psi}_{n}(s)+\frac{n !}{s^{n}\left(e^{s}-1\right)}
$$

Proof. Convergence is clear. Also, by (11),

$$
\begin{aligned}
\hat{\phi}_{n}(s) & =\sum_{k=0}^{\infty} \int_{k}^{k+1} e^{-s t} \phi_{n}(t) d t=\sum_{k=0}^{\infty} e^{-k s} \int_{0}^{1} e^{-s u} \phi_{n}(u+k) d u \\
& =\sum_{k=0}^{\infty} e^{-k s} \int_{0}^{1} e^{-s u}\left(\phi_{n}(u)+n \sum_{j=0}^{k-1}(u+j)^{n-1}\right) d u \\
& =\sum_{k=0}^{\infty} \int_{0}^{1} e^{-s(u+k)} \psi_{n}(u+k) d u+n \sum_{j=0}^{\infty} \sum_{m=j}^{\infty} e^{-(m+1) s} \int_{0}^{1} e^{-s u}(u+j)^{n-1} d u \\
& =\sum_{k=0}^{\infty} \int_{k}^{k+1} e^{-s t} \psi_{n}(t) d t+\frac{n}{e^{s}-1} \sum_{j=0}^{\infty} \int_{j}^{j+1} e^{-s v} v^{n-1} d v \\
& =\int_{0}^{\infty} e^{-s t} \psi_{n}(t) d t+\frac{n \Gamma(n)}{\left(e^{s}-1\right) s^{n}} .
\end{aligned}
$$

LEMMA 5. For $\operatorname{Re}(\lambda)>0$ and $n \geqslant 2$,

$$
1+\frac{\lambda^{n}}{n !} \hat{\phi}_{n}(\lambda)=\frac{1}{\lambda}+\frac{1}{2}+\sum_{j=1}^{\left[\frac{1}{2}(n-1)\right]} \frac{(-1)^{j-1} B_{j} \lambda^{2 j-1}}{(2 j) !} .
$$

Proof. For the power function $f(t)=t^{p}, p \in \mathbb{N}$, we have $\hat{f}(\lambda)=p ! \lambda^{-p-1}$; so (13) follows on taking the Laplace transform of $\phi_{n}$ in (9).

Notice that (13) gives a formula for an arbitrary partial sum of the righthand side of (8). Then (12) immediately gives a formula using $\hat{\psi}_{n}$ for the remainder after finitely many terms:

$$
\frac{e^{\lambda}}{e^{\lambda}-1}=\frac{1}{\lambda}+\frac{1}{2}+\sum_{j=1}^{\left[\frac{1}{2}(n-1)\right]} \frac{(-1)^{j-1} B_{j} \lambda^{2 j-1}}{(2 j) !}-\frac{\lambda^{n} \hat{\psi}_{n}(\lambda)}{n !}
$$

for all $\operatorname{Re}(\lambda)>0, n \geqslant 2$. These two lemmas are crucial. 
2.4 Further notation. For Banach spaces $X$ and $Y, \mathscr{B}(X, Y)$ denotes the Banach space of all bounded linear operators from $X$ into $Y$, with identity $I ; \mathscr{B}(X)$ denotes $\mathscr{B}(X, X)$. For $T \in \mathscr{B}(X, Y), R(\lambda, T)$ denotes the resolvent operator $(\lambda I-T)^{-1}$, an element of $\mathscr{B}(Y, X)$, and $\operatorname{Sp}(T), \operatorname{PtSp}(T), \operatorname{Res}(T)$ denote the spectrum, point spectrum and resolvent set of $T$ respectively.

\section{The standard summation operator $S_{\omega}$}

For any function $x: \mathbb{R}^{+} \rightarrow X$ and $\omega>0, t \geqslant 0$ we define $\left(S_{\omega} x\right)(t)$ by (1),

$$
\left(S_{\omega} x\right)(t)=\sum_{r=0}^{[t / \omega]} x(t-r \omega),
$$

so that also $S_{\omega} x: \mathbb{R}^{+} \rightarrow X . S_{\omega}$ can be thought of as a linear operator which maps any function to a sum of pulses, each the shape of that function, recurring at time intervals $\omega$, starting at time 0 and repeated indefinitely. If $x(u)$ for $u<0$ is interpreted always as 0 then the sum can be written as $\sum_{r=0}^{\infty} x(t-r \omega)$. If $x$ is continuous then $S_{\omega} x$ is continuous if and only if $x(0)=0$.

LeMMA 6. If the Laplace integral $\hat{x}(s)$ converges absolutely and $\operatorname{Re}(s)>0$, then the integral $\left(S_{\omega} x\right)^{n}(s)$ also converges absolutely, and

$$
\left(S_{\omega} x\right)^{\wedge}(s)=\frac{e^{\omega s}}{e^{\omega s}-1} \hat{x}(s) .
$$

Proof.

$$
\begin{aligned}
\left(S_{\omega} x\right)^{\wedge}(s) & =\sum_{j=0}^{\infty} \int_{j \omega}^{(j+1) \omega} e^{-s t} \sum_{r=0}^{j} x(t-r \omega) d t \\
& =\sum_{r=0}^{\infty} \sum_{j=r}^{\infty} \int_{j \omega}^{(j+1) \omega} e^{-s t} x(t-r \omega) d t \\
& =\sum_{r=0}^{\infty} \int_{r \omega}^{\infty} e^{-s t} x(t-r \omega) d t \\
& =\sum_{r=0}^{\infty} \int_{0}^{\infty} e^{-s(u+r \omega)} x(u) d u \\
& =e^{\omega s}\left(e^{\omega s}-1\right)^{-1} \hat{x}(s) .
\end{aligned}
$$

The assumptions on $\hat{x}(s)$ and $s$ are sufficient to justify the change of order at (17), and also to establish the absolute convergence of the integral $\left(S_{\omega} x\right)^{\wedge}(s)$.

We return to discuss $S_{\omega}$ further in Section 5 below. 


\section{The Euler-Maclaurin formula}

We introduce the Euler-Maclaurin operator $E M_{n, \omega}$ by the definition

$$
\begin{aligned}
\left(E M_{n, \omega} x\right)(t)= & \sum_{r=0}^{[t / \omega]} x(t-r \omega)-\frac{1}{\omega} \int_{0}^{t} x(u) d u-\frac{1}{2} x(t) \\
& -\sum_{j=1}^{\left[\frac{1}{2}(n-1)\right]} \frac{(-1)^{j-1} B_{j}}{(2 j) !} \omega^{2 j-1} x^{(2 j-1)}(t) \\
& +\frac{\omega^{n-1}}{n !} \int_{0}^{t} \psi_{n}\left(\frac{t-u}{\omega}\right) x^{(n)}(u) d u .
\end{aligned}
$$

The proof of the Euler-Maclaurin formula, which says that $E M_{n, \omega} x=0$, is an easy deduction from (14) and Lemmas $6,2,1$ and 3. Let $x$ be given, say in $\varsigma_{0}^{n}$. Write $\lambda=\omega s$ in (14) and multiply by $\hat{x}(s)$ : the resulting equation asserts, in view of those lemmas and the fact that $x$ and its derivatives vanish at 0 , that

$$
\left(E M_{n, \omega} x\right)^{\wedge}(s)=0 \text { for } \operatorname{Re}(s)>\max \{0, \rho\} .
$$

Thus $E M_{n, \omega} x$, being a continuously differentiable function whose Laplace transform vanishes on a right halfplane, must vanish identically ([10], p. 61; [4], p. 216 ). Taking $n=2 N$ for simplicity (and noting that the property $x^{(2 N)}(0)=0$ is not used in the proof) we can state the result thus:

THEOREM 1. If $x: \mathbb{R}^{+} \rightarrow X$ and its first $2 N$ derivatives are continuous on $\mathbb{R}^{+}$, and for some positive constants $c$ and $\rho$ satisfy $\left\|x^{(j)}(t)\right\| \leqslant c e^{\rho t}($ all $t \geqslant 0 ; j=$ $0,1, \ldots, 2 N)$, and if $x, x^{\prime}, \ldots, x^{(2 N-1)}$ all vanish at 0 , then

$$
\begin{aligned}
\sum_{r=0}^{[t / \omega]} x(t-r \omega)= & \frac{1}{\omega} \int_{0}^{t} x(u) d u+\frac{1}{2} x(t) \\
& +\sum_{j=1}^{N-1} \frac{(-1)^{j-1} B_{j} \omega^{2 j-1}}{(2 j) !} x^{(2 j-1)}(t) \\
& -\frac{\omega^{2 N-1}}{(2 N) !} \int_{0}^{t} \psi_{2 N}\left(\frac{t-u}{\omega}\right) x^{(2 N)}(u) d u,
\end{aligned}
$$

for all $t \geqslant 0$ and all $\omega>0$.

COROLLARY 1. On $\mathfrak{E}_{0}^{m}$ the operator $E M_{n, \omega}$ is zero, for all $n \leqslant m \leqslant \infty$. 
COROLlaRY 2. Under the assumptions in Theorem 1 , for integers $k, m$ with $0 \leqslant k<m$ we have

$$
\begin{aligned}
\sum_{r=k+1}^{m} x(r \omega)= & \frac{1}{\omega} \int_{k \omega}^{m \omega} x(u) d u+\frac{1}{2}(x(m \omega)-x(k \omega)) \\
& +\sum_{j=1}^{N-1} \frac{(-1)^{j-1} B_{j} \omega^{2 j-1}}{(2 j) !}\left(x^{(2 j-1)}(m \omega)-x^{(2 j-1)}(k \omega)\right) \\
& -\frac{\omega^{2 N-1}}{(2 N) !} \int_{k \omega}^{m \omega} \psi_{2 N}\left(m-\frac{u}{\omega}\right) x^{(2 N)}(u) d u .
\end{aligned}
$$

Here the periodicity of $\psi_{2 N}$ has been used to simplify the remainder term.

Since equation (20) uses valu $s$ of $x$ and its derivatives only in the interval $[k \omega, m \omega]$, whereas the proof uses values on all of $\mathbb{R}^{+}$, we need to show that this dependence upon a wider domain is illusory.

Lemma 7. Let $y:[a, b] \rightarrow X$, where $0<a<b<\infty$, have continuous derivatives $y^{\prime}, y^{\prime \prime}, \ldots, y^{(n)}$ on $[a, b]$; here $n<\infty$. Then there exists a function $x \in \mathbb{\Xi}_{0}^{n}$ such that $x \mid[a, b]=y$.

Proof. For $0 \leqslant t \leqslant a$ take

$$
x(t)=t^{n+1} c_{0}+t^{n+2} c_{1}+\cdots+t^{2 n+1} c_{n}
$$

with coefficients $c_{j}$ in $X$. Clearly $x^{(j)}(0)=0$ for $j=0,1, \ldots, n$; and we can solve the equations $x^{(j)}(a)=y^{(j)}(a)$ for the elements $c_{j}$ uniquely. This extends appropriately the definition of $y$ to the interval $[0, a]$. We deal similarly with the interval $[b, b+1]$ and take $x(t)=0$ for all $t \geqslant b+1$.

From this and the previous corollary we deduce

THEOREM 2. If $k, m \in \mathbb{N}, 0<k<m, \omega>0, N \geqslant 1$ and if $x:[k \omega, m \omega] \rightarrow X$ has continuous derivatives up to and including $x^{(2 N)}$ on $[k \omega, m \omega]$, then (20) holds.

This establishes the Euler-Maclaurin formula almost in the sought generality, by this method of proof. In Theorem 2 we assume $k>0$, so that 0 is not in the domain of $x$, and we next consider how to deal with the case where $x \in \mathbb{E}^{n} \backslash \mathfrak{c}_{0}^{n}$, which covers the case $k=0$.

For any $y$ in $\mathbb{C}^{n}$ there is a unique polynomial $p$ of degree at most $n$ such that the equation $x(t)=y(t)-p(t)$ defines a function $x$ in $\mathbb{S}_{0}^{n}$ : namely, $p$ is the $(n+1)$ th partial sum of the Taylor series of $y$ about 0 . By Theorem 1 we have $E M_{n, \omega} x=0$, so

$$
E M_{n, \omega} y=E M_{n, \omega} P
$$


We introduce the Euler-Maclaurin functional $\Lambda_{n, \omega, m}$ on $\mho^{n}$ (here $m$ is a nonnegative integer) by writing

$$
\Lambda_{n, \omega, m}(y)=\left(E M_{n, \omega} p\right)(m \omega)=\left(E M_{n, \omega} y\right)(m \omega) .
$$

$\Lambda_{n, \omega, m}$ is a linear functional (cf. Hardy's introduction of the 'Euler-Maclaurin constant' of a function, [3, p. 327]). Direct calculation shows, for the polynomials $t^{j}$ and with $n=2 N$ again, that

$$
\text { (23) } E M_{2 N, \omega}\left(t^{j}\right)(m \omega)= \begin{cases}\frac{(-1)^{\frac{1}{2}(j+1)}}{j+1} B_{\frac{1}{2}(j+1)} \omega^{j} & (j \text { odd, } 1 \leqslant j \leqslant 2 N-3), \\ 0 & (j=2 N-1), \\ 0 & (j \text { even, } 2 \leqslant j \leqslant 2 N), \\ \frac{1}{2} & (j=0) .\end{cases}
$$

The numbers on the right are independent of $m$ (and for $2 N \geqslant j+2$ they are also independent of $N$ ). If $p$ is the polynomial of degree $2 N$ constructed as above for $y$ in $\mathbb{E}^{2 N}$ then we get

$$
\Lambda_{2 N, \omega, m}(y)=\left(E M_{2 N, \omega} p\right)(m \omega)=\frac{1}{2} y(0)+\sum_{j=1}^{N-1} \frac{(-1)^{j-1} B_{j} \omega^{2 j-1}}{(2 j) !} y^{(2 j-1)}(0) ;
$$

so $\Lambda$ is independent of $m$, and its third suffix can be dropped. Therefore $\left(E M_{2 N, \omega} y\right)(m \omega)$ is equal to this expression on the right in (24). This equation, on examination, turns out to be nothing other than equation (20) with $x$ replaced by $y$ and with $k$ put equal to 0 . Thus

THEOREM 3. Theorem 2 holds also when $k=0$. This is so whether or not $x$ and its derivatives vanish at 0 .

While $\left(E M_{2 N, \omega} x\right)(t)=0$ for all $t \geqslant 0$ when $x \in \mathbb{E}_{0}^{2 N}$, we do not assert the same identity when $x \in \mathbb{C}^{2 N}$; we have shown only that $E M_{2 N, \omega} x$ then takes the same values at all integer multiples of $\omega$.

\section{Further properties of $S_{\omega}$}

5.1 Each $S_{\omega}$ is a Baxter operator with parameter value 1 , in the sense that it satisfies the identity

$$
S_{\omega} x \cdot S_{\omega} y=S_{\omega}\left(S_{\omega} x \cdot y+x \cdot S_{\omega} y-x \cdot y\right)
$$

for all functions $x, y$ on $\mathbb{R}^{+}$, using pointwise multiplication. The property cannot be formulated in $\mathbb{C}^{n}$ when $\rho>0$ since the products are not well defined there; 
however (25) is certainly meaningful for arbitrary functions on $\mathbb{R}^{+}$. Identity (25) is the starting point of the analysis in [7].

THEOREM 4. For every $\omega>0$ and $0 \leqslant n \leqslant \infty, S_{\omega}$ is a bounded linear operator on $\mathfrak{C}_{0}^{n}\left(\mathbb{R}^{+}, X ; \rho\right)$ with norm

$$
\left|S_{\omega}\right|_{n} \leqslant e^{\rho \omega}\left(e^{\rho \omega}-1\right)^{-1},
$$

provided $\rho>0$.

Proof. Let $n$ be finite, and $x \in \mathfrak{E}_{0}^{n}$. If $k \omega<t<(k+1) \omega$ then for $j=$ $0,1,2, \ldots, n$,

$$
\left(S_{\omega} x\right)^{(j)}(t)=\sum_{r=0}^{k} x^{(j)}(t-r \omega) .
$$

Calculation shows that the same formula holds when $t=k \omega$ because $x$ and its derivatives vanish at 0 , and that $\left(S_{\omega} x\right)^{(j)}$ is continuous. (In order for $\left(S_{\omega} x\right)^{(n)}(t)$ to exist everywhere we must assume $x^{(n)}(0)=0$. This is the reason for requiring $x^{(n)}(0)=0$ in the definition of $\mathbb{E}_{0}^{n}$, although the property was not needed for Theorem 1.) Since $S_{\omega} x(t)=x(t)$ for $0 \leqslant t<\omega,\left(S_{\omega} x\right)^{(j)}(0)=0$ for $j=0,1, \ldots, n$. Finally, when $k \omega \leqslant t<(k+1) \omega$ we have, by (7),

$$
\left\|S_{\omega} x^{(j)}(t)\right\| \leqslant \sum_{r=0}^{k}\left\|x^{(j)}(t-r \omega)\right\| \leqslant \sum_{r=0}^{k}[x]_{j} e^{\rho(t-r \omega)} \leqslant[x]_{j} e^{\rho t} \sum_{r=0}^{\infty} e^{-r \rho \omega}
$$

so $\left|S_{\omega} x\right|_{n}=\sum_{j=0}^{n} \sup _{t \geqslant 0} \|\left(S_{\omega} x\right)^{(j)}(t) e^{-\rho t}|| / j ! \leqslant e^{\rho \omega}\left(e^{\rho \omega}-1\right)^{-1}|x|_{n}$. The result follows.

Note that $S_{\omega}$ does not map $\mathbb{S}^{n}$ into $\mathbb{C}^{n}$, or even into $\mathbb{C}^{0}$. It is clear also that the proof fails for $\rho \leqslant 0$, and hence for the algebras $\mathbb{C}_{0}^{n}(\mathrm{P})$ of Section 2.2. Henceforth we assume $\rho>0$.

THEOREM 5. For all $\omega>0$ and $0 \leqslant n \leqslant \infty$ the point spectrum of $S_{\omega}$ as an element of $\mathscr{B}\left(\mathbb{E}_{0}^{n}\right)$ is empty, and 1 belongs to the residual spectrum. When $\lambda \neq 1$,

$$
\left(\lambda I-S_{\omega}\right)^{-1} x(t)=\frac{1}{\lambda-1} x(t)+\frac{1}{(\lambda-1)^{2}} \sum_{r=1}^{[t / \omega]}\left(\frac{\lambda}{\lambda-1}\right)^{r-1} x(t-r \omega)
$$

for all $t \geqslant 0$ (the sum is zero when $0 \leqslant t<\omega$ ). In particular, $0 \in \operatorname{Res}\left(S_{\omega}\right)$ and

$$
S_{\omega}^{-1} x(t)=x(t) \quad(0 \leqslant t<\omega), \quad x(t)-x(t-\omega) \quad(t \geqslant \omega) .
$$

When $\lambda=1$ we have

(30) $\operatorname{dom}\left(\left(I-S_{\omega}\right)^{-1}\right)=\left\{x \in \mathfrak{S}_{0}^{n}: x(t)=0\right.$ for $0 \leqslant t \leqslant \omega$,

$$
\text { and } \left.x^{(j)}(\omega)=0 \text { for } j=1,2, \ldots, n\right\},
$$




$$
\left(I-S_{\omega}\right)^{-1} x(t)=x(t)-x(t+\omega) \quad(t \geqslant 0),
$$

and $\left(I-S_{\omega}\right)^{-1}$ is a bounded operator on its domain. For all $n$,

$$
\operatorname{Sp}\left(S_{\omega}\right) \subseteq\left\{\lambda:\left|\lambda(\lambda-1)^{-1}\right| \geqslant e^{\rho \omega}\right\}
$$

(the righthand side is a closed disc), and the resolvent $R\left(\lambda, S_{\omega}\right)$ is given by (28) when $\lambda$ is not in $\operatorname{Sp}\left(S_{\omega}\right)$. When $n$ is finite there is equality in (32).

Proof. Straightforward calculations from the definition (15) show that for all $\lambda,\left(\lambda I-S_{\omega}\right) y=0$ implies $y=0$, so $\operatorname{PtSp}\left(S_{\omega}\right)=\varnothing$. Moreover, for any $y \in \mathbb{E}_{0}^{n}$, $\left(I-S_{\omega}\right) y$ belongs to the righthand side of (30). Conversely, if $x$ belongs to the righthand side then (31) and boundedness are easily verified, and this completes the proof of (30).

To prove (28), write the righthand side as $V x(t)$; it can be verified that $\left(\lambda I-S_{\omega}\right) V x=V\left(\lambda I-S_{\omega}\right) x=x$ and so (28) holds for all $x$ in the domain of $V$.

The previous proposition gives an upper bound for $\left|S_{\omega}\right|_{n}$ and hence for the spectral radius of $S_{\omega}$. To get the sharper result (32), write $y(t)=V x(t)$; for $k \omega \leqslant t \leqslant(k+1) \omega$ we have

$$
\left\|y^{(j)}(t)\right\| \leqslant \frac{1}{|\lambda-1|}\left\|x^{(j)}(t)\right\|+\frac{1}{|\lambda-1|^{2}} \sum_{r=1}^{k}\left|\frac{\lambda}{\lambda-1}\right|^{r-1}\left\|x^{(j)}(t-r \omega)\right\|
$$

so

$$
[y]_{j} \leqslant \frac{1}{|\lambda-1|}\left\{1+\frac{1}{|\lambda|} \sum_{r=1}^{\infty}\left(\left|\frac{\lambda}{\lambda-1}\right| e^{-\rho \omega}\right)^{r}\right\}[x]_{j}
$$

and therefore, with $n \leqslant \infty$,

$$
|y|_{n} \leqslant|\lambda-1|^{-1}\left\{1+\left(|\lambda-1| e^{\rho \omega}-|\lambda|\right)^{-1}\right\}|x|_{n}
$$

provided $\left|\lambda(\lambda-1)^{-1}\right|<e^{\rho \omega}$. Thus for all such numbers $\lambda, V \in \mathscr{B}\left(\mathbb{S}_{0}^{n}\right)$ and so $\lambda \in \operatorname{Res}\left(S_{\omega}\right)$. This proves the inclusion.

Now suppose that $n$ is finite. Let $x$ be a function which equals $e^{\rho t} a$ for $t \geqslant \omega$ ( $a$ being any nonzero element of $X$ ) and is defined on $[0, \omega]$ in such a way that $x \in \mathbb{E}_{0}^{n}\left(x\right.$ can be constructed as in the proof of Lemma 7). If $\lambda(\lambda-1)^{-1}=e^{\rho \omega_{z}}$ we have, for $k \omega \leqslant t<(k+1) \omega$ and $|z|>1$,

$$
y(t) e^{-\rho t}=\frac{1}{\lambda-1}+\frac{e^{-\rho \omega}\left(z^{k}-1\right)}{(\lambda-1)^{2}(z-1)},
$$

and with fixed $\lambda$ this is unbounded as $k \rightarrow \infty$. So for such $\lambda,[y]_{0}=+\infty$, $y \notin \mathbb{E}_{0}^{n}$. Therefore if $\lambda$ is in the open disc $\left|\lambda(\lambda-1)^{-1}\right|>e^{\rho \omega}$ then $x$ does not belong to the domain of $\left(\lambda I-S_{\omega}\right)^{-1}$, and so $\lambda \in \operatorname{Sp}\left(S_{\omega}\right)$; since the spectrum is closed there must be equality in (32). 
COROllary. For finite $n,\left|S_{\omega}\right|_{n}=e^{\rho \omega}\left(e^{\rho \omega}-1\right)^{-1}$.

Proof. The result just proved shows that the spectral radius is $e^{\rho \omega}\left(e^{\rho \omega}-1\right)^{-1}$, and the norm is not less than this; nor more, by (26).

The integration operator $K$ of $(5)$ belongs to $\mathscr{B}\left(\mathbb{C}^{n}\right)$ and maps $\mathbb{C}_{0}^{n}$ into $\mathfrak{C}_{0}^{n}$ with norm $|K|_{n} \leqslant 1+1 / \rho$. For $n \leqslant \infty$ it is quasinilpotent as an element of $\mathscr{B}\left(\mathfrak{S}_{0}^{n}\right)$.

The differentiation operator $D$, for which

$$
(D x)(t)=x^{\prime}(t), \quad \operatorname{dom}(D)=\left\{x \in \mathbb{E}_{0}^{n}: x^{\prime} \in \mathfrak{S}_{0}^{n}\right\},
$$

is closed, unbounded with dense domain; in fact $\operatorname{dom}(D)=C_{0}^{n+1}$ (see Section 5.2 , below). The point spectrum of $D$ is empty and $\operatorname{Sp}(D)$ is included in the right halfplane $\operatorname{Re}(\lambda) \geqslant \rho$. In particular, $0 \in \operatorname{Res}(D)$ so $D^{-1}$ is bounded on $\mathbb{E}_{0}^{n}$; in fact $D^{-1}=K$. On heuristic grounds we would expect that $S_{\omega}$ and $D$ are related by

$$
S_{\omega}=e^{\omega D}\left(e^{\omega D}-I\right)^{-1}
$$

(see [7], equation (52); but note that in the present circumstances the righthand side of (34) is not defined), and hence we would expect that $\operatorname{Sp}\left(S_{\omega}\right)=$ $\left\{e^{\omega \lambda}\left(e^{\omega \lambda}-1\right)^{-1}: \lambda \in \operatorname{Sp}(D)\right\}$. In this connection note that the function $\phi(\lambda)=$ $e^{\lambda}\left(e^{\lambda}-1\right)$ maps the halfplane $\operatorname{Re}(\lambda) \geqslant \rho$ onto the $\operatorname{disc}\left|\mu(\mu-1)^{-1}\right| \geqslant e^{\rho \omega}$ of (32). Theorem 5 therefore suggests that $\operatorname{Sp}(D)$ is precisely the halfplane when $n$ is finite; but we lack a proof of this.

The operator $R_{\omega, N}$ giving the remainder in (19),

$$
\left(R_{\omega, N} x\right)(t)=-\frac{\omega^{2 N-1}}{(2 N) !} \int_{0}^{t} \psi_{2 N}\left(\frac{t-u}{\omega}\right) x^{(2 N)}(u) d u,
$$

is closed unbounded on $\mathbb{E}_{0}^{2 N}$, since

$$
S_{\omega}=\frac{1}{\omega} K+\frac{1}{2} I+\sum_{j=1}^{N-1} \frac{(-1)^{j-1} B_{j} \omega^{2 j-1}}{(2 j) !} D^{2 j-1}+R_{\omega, N} .
$$

5.2 Instead of working in $\mathbb{C}_{0}^{n}$ for a fixed $n$ we can treat $K$ and $D$ as maps between such spaces; the picture is then simpler. In what follows we assume always that $n$ is finite, and $\mathbb{S}_{0}^{n}$ denotes the space with its own norm $\mid \cdot l_{n}$ whereas $C_{0}^{n}$ will stand for $\mathbb{E}_{0}^{n}$ as a dense subspace of $\mathbb{E}_{0}^{m}$ for some $m<n$, so that $C_{0}^{n}$ carries the relativized norm of $\mathbb{S}_{0}^{m}$. The norm of $\mathscr{B}\left(\mathbb{S}_{0}^{m}, \mathbb{S}_{0}^{n}\right)$ will be written $|\cdot|_{m, n}$. We have seen that, for $K, D$ and $S_{\omega}$ mapping from $\mathbb{E}_{0}^{n}$ into $\mathbb{E}_{0}^{n}$,

$$
\operatorname{ran}(K)=\operatorname{dom}(D)=C_{0}^{n+1},
$$

and $K$ and $S_{\omega}$ bounded, $D$ unbounded. 
THEOREM 6. The injection maps id: $\mathbb{C}_{0}^{n+1} \rightarrow \mathbb{E}_{0}^{n}$ and id: $\mathbb{C}_{0}^{\infty} \rightarrow \mathbb{E}_{0}^{n}$ are bounded. The maps $K: \mathbb{E}_{0}^{n} \rightarrow \mathbb{E}_{0}^{n+1}, D: \mathbb{E}_{0}^{n+1} \rightarrow \mathbb{E}_{0}^{n}$ are bounded isomorphisms of vector spaces, each the inverse of the other, with norms

$$
|K|_{n, n+1} \leqslant \max \{1,1 / \rho\}, \quad|D|_{n+1, n} \leqslant n+1 ;
$$

the operators $K, D$ and $S_{\omega}: \mathbb{E}_{0}^{n} \rightarrow \mathbb{E}_{0}^{n}$ commute pairwise.

The proof is straightforward. The statement about commutativity must be suitably interpreted: for $D$ and $S_{\omega}$ for example it says that $S_{\omega} D=D S_{\omega}$ as operators from $\mathbb{E}_{0}^{n+1}$ onto $\mathbb{C}_{0}^{n}$.

5.3 We turn to some properties of the function

$$
S: \omega \mapsto S_{\omega}, \quad \mathbb{R}^{+} \backslash\{0\} \rightarrow \mathscr{B}\left(\mathbb{S}_{0}^{n}\right)
$$

and the family $\left(S_{\omega}\right)_{\omega>0}$, supposing always that $\rho>0$ and $n$ is finite. First, an addition law:

\section{LEMMA 8. For all positive $\alpha$ and $\beta$,}

$$
S_{\alpha+\beta}\left(S_{\alpha}+S_{\beta}-I\right)=S_{\alpha} S_{\beta},
$$

and $S_{\alpha}$ and $S_{\beta}$ commute.

Proof. For $x \in \mathfrak{S}_{0}^{n}$ and $t \geqslant 0$,

$$
S_{\alpha} S_{\beta} x(t)=\sum_{j=0}^{[t / \alpha]} \sum_{k=0}^{[(t-j \alpha) / \beta]} x(t-j \alpha-k \beta) .
$$

Treating $j$ and $k$ as a pair of real variables, let $\Delta$ denote the closed triangular region in $\mathbb{R}^{2}$ bounded by the lines $j=0, k=0$ and $j \alpha+k \beta=t$. The righthand side of $(40)$ is the sum of terms $x(t-j \alpha-k \beta)$ taken over all lattice points $(j, k)$ in $\Delta$. Using such representations for each of the terms in (39), we find that the proof of (39) reduces to making a simple partition of $\Delta$ into two triangles having a common side. Commutativity of the range of $S$ follows immediately from (39).

Identity (39) is implicit in the heuristic formula (34). It can be iterated: for example, $S_{\alpha+\beta+\gamma}\left(S_{\beta} S_{\gamma}+S_{\gamma} S_{\alpha}+S_{\alpha} S_{\beta}-S_{\alpha}-S_{\beta}-S_{\gamma}+I\right)=S_{\alpha} S_{\beta} S_{\gamma}$. Perhaps the most illuminating view of (39) comes by introducing the operators

$$
T_{\omega}=S_{\omega}\left(S_{\omega}-I\right)^{-1}=\left(I-S_{\omega}^{-1}\right)^{-1}
$$

with domains (30); we find that $T_{\omega} x(t)=x(t+\omega)$, so $T_{\omega}$ is the restriction to its domain of backward translation, and (39) becomes the semigroup property $T_{\alpha+\beta}=T_{\alpha} T_{\beta}$. But notice that $\operatorname{dom}\left(T_{\omega}\right)$ depends upon $\omega$.

Two important questions about the function $S$ concern, in one form or another,

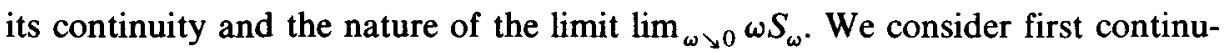
ity, and also differentiability. 
5.4 If $x \in \mathbb{C}_{0}^{n}$ then the $X$-valued function $(\omega, t) \mapsto S_{\omega} x(t)$ is easily shown to be continuous: one considers separately the cases where $t / \omega$ is or is not an integer. The continuity of the functions $\omega \rightarrow S_{\omega} x$ and $\omega \mapsto S_{\omega}$ is more delicate. First, we give an easy result in which $S_{\omega}$ is allowed to map into a larger space.

THEOREM 7. For all finite $n \geqslant 1$ the function $\omega \rightarrow S_{\omega},(0, \infty) \rightarrow \mathscr{B}\left(\mathbb{E}_{0}^{n}, \mathbb{E}_{0}^{n-1}\right)$ is norm-continuous, uniformly with respect to $\omega$ on any halfline $[\eta, \infty)$ with $\eta>0$.

Proof. For given $x \in \Subset_{0}^{n}, \omega>0, t \geqslant 0$, small $\varepsilon$, write

$$
y_{e}(t)=S_{\omega+\varepsilon} x(t)-S_{\omega} x(t) .
$$

The meanvalue theorem gives

$$
\left\|y_{\varepsilon}^{(l)}(t)\right\| \leqslant 2|\varepsilon|[x]_{l+1} e^{\rho t} \sum_{j=0}^{\infty} j e^{-j \rho \omega} \quad(l=0,1, \ldots, n-1)
$$

(but there is no inequality for $l=n$ ). Write

$$
g_{p}(\alpha)=\sum_{j=0}^{\infty} j^{p} e^{-j \alpha}, \quad \text { so that } g_{1}(\alpha)=e^{\alpha}\left(e^{\alpha}-1\right)^{-2} ;
$$

then

$$
\begin{gathered}
\left|y_{e}\right|_{n-1} \leqslant 2 n g_{1}(\omega)|\varepsilon||x|_{n}, \\
\left|S_{\omega+\varepsilon}-S_{\omega}\right|_{n, n-1} \leqslant \frac{2 n e^{\rho \omega}}{\left(e^{\rho \omega}-1\right)^{2}}|\varepsilon| .
\end{gathered}
$$

The theorem follows.

To $S_{\omega}$ there also corresponds an operator $U_{\omega}$ recognizable as its derivative, namely

$$
\left(U_{\omega} x\right)(t)=-\sum_{r=1}^{[t / \omega]} r x^{\prime}(t-r \omega) ;
$$

however, to establish that $U_{\omega}=d S_{\omega} / d \omega$ we have to work in $\mathscr{B}\left(\mathfrak{C}_{0}^{n}, \mathbb{C}_{0}^{n-2}\right)$.

THEOREM 8. Let $1 \leqslant n<\infty$. If $x \in \mathbb{S}_{0}^{n}$ then $U_{\omega} x$, defined by (47), belongs to $\mathfrak{S}_{0}^{n-1}$, and $U_{\omega} \in \mathscr{B}\left(\mathfrak{C}_{0}^{n}, \mathfrak{C}_{0}^{n-1}\right)$,

$$
\mid U_{\omega, n-1} \leqslant n g_{1}(\rho \omega) \text {. }
$$

If $n \geqslant 2$ the function $S:(0, \infty) \rightarrow \mathscr{B}\left(\mathbb{C}_{0}^{n}, \mathfrak{C}_{0}^{n-2}\right)$ is differentiable and

$$
\frac{d S_{\omega}}{d \omega}=U_{\omega}
$$


Proof. Let $x \in \mathbb{S}_{0}^{n}$. It is easily verified that for fixed $t$ the $X$-valued function $f$ : $\omega \mapsto S_{\omega} x(t)$ is differentiable and $f^{\prime}(\omega)=U_{\omega} x(t)$ for all $\omega>0$. Using the meanvalue theorem as in the proof of Theorem 7 we can show that $z$, defined for fixed $\omega$ by $z(t)=\left(U_{\omega} x\right)(t)$, satisfies $|z|_{n-1} \leqslant n g_{1}(\rho \omega)|x|_{n}$; this proves (48) and the preceding statement. Now write, for small $\varepsilon$,

$$
v_{\varepsilon}(t)=\varepsilon^{-1} y_{\varepsilon}(t)-z(t)=\left(\varepsilon^{-1}\left(S_{\omega+\varepsilon} x-S_{\omega} x\right)-z\right)(t) .
$$

Two applications of the mean-value theorem give $\left.\left[v_{\varepsilon}\right]_{l} \leqslant 2 g_{2}(\rho \omega) \mid \varepsilon \| x\right]_{l+2}$,

$$
\left|\varepsilon^{-1}\left(S_{\omega+\varepsilon}-S_{\omega}\right)-U_{\omega}\right|_{n, n-2} \leqslant 2 n^{2} g_{2}(\rho \omega)|\varepsilon| .
$$

Then (49) follows; moreover differentiation is uniform on $[\eta, \infty)$ when $\eta>0$.

More generally, if $1 \leqslant p \leqslant n-1$ we write

$$
U_{\omega, p} x(t)=(-1)^{p} \sum_{r=1}^{[t / \omega]} r^{p} x^{(p)}(t-r \omega), \quad U_{\omega, 1}=U_{\omega}
$$

then

$$
U_{\omega, p} \in \mathscr{B}\left(\mathbb{E}_{0}^{n}, \mathbb{E}_{0}^{n-p}\right), \quad\left|U_{\omega, p}\right|_{n, n-p} \leqslant\left(\begin{array}{l}
n \\
p
\end{array}\right) g_{p}(\rho \omega) ;
$$

and in $\mathscr{B}\left(\mathbb{E}_{0}^{n}, \mathbb{\mho}_{0}^{n-p-1}\right)$ we have

$$
\frac{d^{p} S_{\omega}}{d \omega^{p}}=U_{\omega, p}
$$

We remark that $g_{p}(\alpha)$ has the form $e^{\alpha}\left(e^{\alpha}-1\right)^{-p-1} Q_{p-1}\left(e^{\alpha}\right)$ where $Q_{p-1}(t)$ is a monic polynomial of degree $p-1 ; Q_{0}(t)=1, Q_{1}(t)=t+1, Q_{2}(t)=t^{2}+4 t+$ $1, \ldots$ For a summation formula for $U_{\omega}$ see Section 6.1 below.

We turn to the continuity of $\omega \mapsto S_{\omega}$ when, as is more natural, $S_{\omega}$ has the role of an operator in $\mathscr{B}\left(\mathbb{C}_{0}^{n}\right)$. Here even strong continuity does not hold on $\mathbb{C}_{0}^{n}$; however it holds on a non-trivial subspace. To prove this we introduce first the subspaces

$$
\begin{aligned}
F^{n}(\alpha)=\left\{x \in \mathbb{E}_{0}^{n}: \text { the function } \omega\right. & \mapsto S_{\omega} x,(0, \infty) \rightarrow \mathbb{E}_{0}^{n}, \\
& \text { is continuous at } \omega=\alpha\} .
\end{aligned}
$$

Because the operators $S_{\omega}$ are uniformly bounded for say $\omega \geqslant \frac{1}{2} \alpha$ (Theorem 4), $F^{n}(\alpha)$ is a closed vector subspace of $\mathfrak{E}_{0}^{n}$; and clearly $F^{n}(\alpha)$ is invariant under any bounded linear operator which commutes with the operators $S_{\omega}$. We shall show that $F^{n}(\alpha)$ is independent of $\alpha$. For this a preliminary lemma is needed.

LemMa 9. For all positive $\alpha, \beta$ with $\alpha \neq \beta$ the operators $S_{\alpha}-S_{\beta}$ and $S_{\alpha}+S_{\beta}-I$ are one-one, and the inverse of each is bounded on its domain. 
Proof. Suppose $\alpha>\beta$. Lemma 8 gives $\left(S_{\alpha}-S_{\beta}\right) S_{\alpha-\beta}=S_{\alpha}\left(I-S_{\beta}\right)$, and therefore since $S_{\alpha-\beta}$ is regular in $\mathscr{B}\left(\mathfrak{C}_{0}^{n}\right)$ by Theorem 5 ,

$$
S_{\alpha}-S_{\beta}=S_{\alpha-\beta}^{-1} S_{\alpha}\left(I-S_{\beta}\right),
$$

and the assertion about $S_{\alpha}-S_{\beta}$ follows from the same theorem. The assertion about $S_{\alpha}+S_{\beta}-I$ is immediate from (39).

LemMA 10. The spaces $F^{n}(\alpha)$ are independent of $\alpha$, that is, if $\omega \mapsto S_{\omega} x$ is continuous for some $\omega$ then it is continuous for all $\omega>0$; moreover the continuity is uniform with respect to $\omega$ in any interval $[\eta, \infty), \eta>0$.

Proof. First we show that $0<\alpha<\beta$ implies $F^{n}(\beta) \subseteq F^{n}(\alpha)$. Let $x \in F^{n}(\beta)$. For $|\varepsilon| \rightarrow 0$ we have, in $\mathbb{C}_{0}^{n}$,

$$
S_{\beta+\varepsilon}\left(S_{\beta-\alpha}+S_{\alpha}-I\right) x-S_{\beta}\left(S_{\beta-\alpha}+S_{\alpha}-I\right) x \rightarrow 0
$$

since all the operators commute. The lefthand side

$$
\begin{aligned}
& =S_{\beta-\alpha+\alpha+\varepsilon}\left(S_{\beta-\alpha}+S_{\alpha+\varepsilon}-I\right) x+S_{\beta+\varepsilon}\left(S_{\alpha}-S_{\alpha+\varepsilon}\right) x-S_{\beta-\alpha} S_{\alpha} x \\
& =S_{\beta-\alpha} S_{\alpha+\varepsilon} x-S_{\beta-\alpha} S_{\alpha} x+S_{\beta+\varepsilon}\left(S_{\alpha}-S_{\alpha+\varepsilon}\right) x \\
& =\left(S_{\alpha+\varepsilon}-S_{\alpha}\right)\left(S_{\beta-\alpha}-S_{\beta+\varepsilon}\right) x .
\end{aligned}
$$

Here we have used Lemma 8. Write $y=\left(S_{\beta-\alpha}-S_{\beta}\right) x$; we get

$$
\left(S_{\alpha+\varepsilon}-S_{\alpha}\right) y+\left(S_{\alpha+\varepsilon}-S_{\alpha}\right)\left(S_{\beta}-S_{\beta+\varepsilon}\right) x \rightarrow 0 .
$$

The second term has norm at most $2\left(1-e^{-\frac{1}{2} \rho \alpha}\right)^{-1}\left|\left(S_{\beta}-S_{\beta+\varepsilon}\right) x\right|_{n}$ by (26), so $\left(S_{\alpha+\varepsilon}-S_{\alpha}\right) y \rightarrow 0, y \in F^{n}(\alpha)$. Lemma 9 says that $\left(S_{\beta-\alpha}-S_{\beta}\right)^{-1}$ is bounded on its domain; since this operator commutes with, and its domain is invariant under, every $S_{\omega}$, it follows that $x \in F^{n}(\alpha)$. This proves $F^{n}(\beta) \subseteq F^{n}(\alpha)$. A similar argument using the other half of Lemma 9 proves the reverse inclusion. A more careful accounting in the proof using the bound (26) shows that the continuity is uniform, as stated.

In view of Lemma 10 we write $F^{n}$ in place of $F^{n}(\alpha)$.

5.5 It is evident from the Euler-Maclaurin series (36) applied to $x$ in $\mathfrak{\mho}_{0}^{n}$, at fixed $t$, that

$$
\lim _{\omega \searrow 0} \omega S_{\omega} x(t)=K x(t) ;
$$

for $\psi_{2 N}$ is bounded and so (35) gives $\lim _{\omega \backslash 0} R_{\omega, N} x(t)=0$. Equation (55) is the assertion that the integral of $x$ on $[0, t]$ is the limit of a net of Riemann sums using 'equipartitions'; more precisely, using the equipartition, for each $\omega$, of the interval $[t-([t / \omega]+1) \omega, t]$ restricted to $[0, t]$, and evaluations of the function at the righthand endpoints. To examine the extent to which the limit exists in 
norm we introduce the subspaces

$$
\begin{gathered}
L^{n}=\left\{x \in \mathbb{C}_{0}^{n}: \lim _{\omega \succ 0} \omega S_{\omega} x \text { exists in } \mathfrak{C}_{0}^{n}\right\}, \\
M^{n}=\left\{y \in \mathbb{S}_{0}^{n}: y=\lim _{\omega \searrow 0} \omega S_{\omega} x \text { in } \mathfrak{S}_{0}^{n}, \text { for some } x \in \mathbb{C}_{0}^{n}\right\} .
\end{gathered}
$$

Since the limit here must be $K x$ when it exists, $y$ determines $x$ uniquely in (57), in fact $x=D y$; and $M^{n}=K\left(L^{n}\right)$. Since the operators $\omega S_{\omega}$ are uniformly bounded as $\omega \searrow 0, L^{n}$ is a closed vector subspace.

THEOREM 9. There are the following relations of inclusion among these subspaces of $\mho_{0}^{n}$, for $n \geqslant 0$ :

$$
C_{0}^{n+2} \subseteq M^{n} \subseteq C_{0}^{n+1} \subseteq F^{n} \cap L^{n}
$$

so none of these subspaces is trivial.

Proof. Since $\operatorname{ran}(K)=C_{0}^{n+1}$ (Theorem 6), we have $M^{n} \subseteq C_{0}^{n+1}$. By property (45), if $x \in C_{0}^{n+1}$ then $x \in F^{n}$, so $C_{0}^{n+1} \subseteq F^{n}$.

To prove $C_{0}^{n+1} \subseteq L^{n}$ we use the Euler-Maclaurin formula (19) or (36) for the case $N=1$. Let $x \in C_{0}^{n+1}$; then

$$
\omega S_{\omega} x=K x+\frac{1}{2} \omega x+z
$$

where

$$
z(t)=\omega R_{\omega, 1} x(t)=-\frac{\omega^{2}}{2} \int_{0}^{t} \psi_{2}\left(\frac{t-u}{\omega}\right) x^{\prime \prime}(u) d u .
$$

To prove $\omega S_{\omega} x \rightarrow K x$ it suffices therefore to show that $\left|\omega R_{\omega, 1}\right|_{n} \rightarrow 0$. Differentiate once with respect to $t$ in (60); we get

$$
z^{\prime}(t)=-\frac{\omega}{2} \int_{0}^{t} \psi_{2}^{\prime}\left(\frac{t-u}{\omega}\right) x^{\prime \prime}(u) d u
$$

since $\psi_{2}(0)=0\left(\phi_{2}(t)=t^{2}-t\right)$. (Since $\psi_{2}^{\prime}(v)$ is not defined at the points $v \in \mathbb{N}$, a full verification of (61) involves partitioning $[0, t]$ into subintervals $[t-(k+1) \omega, t-k \omega]$ and differentiating each such integral.) Now write this as

$$
z^{\prime}(t)=-\frac{\omega}{2} \int_{0}^{t} \psi_{2}^{\prime}\left(\frac{v}{\omega}\right) x^{\prime \prime}(t-v) d v
$$

for $j=1,2, \ldots, n$ we have

$$
z^{(j)}(t)=-\frac{\omega}{2} \int_{0}^{t} \psi_{2}^{\prime}\left(\frac{v}{\omega}\right) x^{(j+1)}(t-v) d v,
$$

and hence

$[z]_{j} \leqslant(\omega / 2 \rho)[x]_{j+1} \quad(j=1,2, \ldots, n), \quad[z]_{j} \leqslant\left(\omega^{2} / 8 \rho\right)[x]_{1} \quad(j=0)$. 
So as $\omega \searrow 0$,

$$
\left|\omega R_{\omega, 1} x\right|_{n} \leqslant(\omega(n+1) / 2 \rho)|x|_{n+1} \rightarrow 0 .
$$

This proves that $C_{0}^{n+1} \subseteq L^{n}$. Finally, this implies $C_{0}^{n+2}=K\left(C_{0}^{n+1}\right) \subseteq K\left(L^{n}\right)=$ $M^{n}$.

W. Walter [11] has pointed out a class of uniformly continuous functions which lie in $F^{n} \cap L^{n}$. Suppose $x \in \mathbb{E}_{0}^{n}$ and there exists a function $\delta: \mathbb{R}^{+} \rightarrow \mathbb{R}^{+}$such that (i) $\left|x-x_{h}\right| \leqslant \delta(h)$ for $h>0$ (here $x_{h}(t)=x(t-h)(t \geqslant h)$, and $x_{h}(t)=0$ $(0 \leqslant t<h)$ ), (ii) $\delta(h) \searrow 0$ as $h \searrow 0$, and (iii) for some $p \in \mathbb{N}, \delta(m h) \leqslant m^{p} \delta(h)$ for all $m \in \mathbb{N}, h>0$. Then $\left|S_{\omega+h} x-S_{\omega} x\right| \leqslant \delta(h) g_{p}(\rho \omega)$ and $\left|\omega S_{\omega} x-K x\right| \leqslant$ $\left(1-e^{-\rho \omega}\right)^{-1} \int_{0}^{\omega} \delta(u) d u$, so $x \in F^{n} \cap L^{n}$.

\section{Other summation formulae}

There are other summation formulae similar to the Euler-Maclaurin formula and associated with summation-type operators rather like $S_{\omega}$. We mention a few of these.

6.1 Theorem 8 gives information about $U_{\omega}=d S_{\omega} / d \omega$. By differentiating (36) with respect to $\omega$ we obtain, for $x \in \mathbb{E}_{0}^{2 N}$,

$$
\begin{aligned}
\sum_{r=1}^{[t / \omega]} r x^{\prime}(t & -r \omega)=\frac{1}{\omega^{2}} K x(t)-\sum_{j=1}^{N-1} \frac{(-1)^{j-1}(2 j-1)}{(2 j) !} B_{j} \omega^{2 j-2} x^{(2 j-1)}(t) \\
& +\frac{\omega^{2 N-2}}{(2 N) !} \int_{0}^{t} x^{(2 N)}(t-u)\left((2 N-1) \psi_{2 N}\left(\frac{u}{\omega}\right)-2 N \frac{u}{\omega} \psi_{2 N-1}\left(\frac{u}{\omega}\right)\right) d u .
\end{aligned}
$$

This formula but without remainder is proved by Laplace transform techniques in a space of generalized functions in [8, equation (58)]. Note that the kernel in the integral is not periodic. Further differentiations give more summation formulae.

6.2 Boole's summation formula. In the spirit of the present discussion this is formulated as

$$
\begin{aligned}
\sum_{r=1}^{[t / \omega]}(-1)^{r-1} x(t-r \omega)= & \frac{1}{2} x(t)+\sum_{j=1}^{N} \frac{(-1)^{j}\left(4^{j}-1\right)}{(2 j) !} B_{j} \omega^{2 j-1} x^{(2 j-1)}(t) \\
& -\frac{\omega^{2 N-1}}{(2 N-1) ! 2} \int_{0}^{t} H_{2 N-1}\left(\frac{t-u}{\omega}\right) x^{(2 N)}(u) d u,
\end{aligned}
$$

where $H_{n}$ is defined below; see B. C. Berndt and L. Schoenfeld [1, p. 31] and [2]. A proof along the lines of Sections 2-4 using the Laplace transformation is based 
on the Euler polynomials $E_{n}(t)$ in place of the Bernoulli polynomials. $E_{n}(t)$ is defined by the generating function

$$
\frac{2 e^{t z}}{e^{z}+1}=\sum_{n=0}^{\infty} \frac{E_{n}(t)}{n !} z^{n} \quad(\text { for }|z|<\pi) .
$$

Let $H_{n}$ be the alternating periodic extension of $E_{n}$ outside $[0,1), H_{n}(t)=$ $(-1)^{[t]} E_{n}(t-[t])$. We find that

$$
\hat{E}_{n}(s)=\hat{H}_{n}(s)+\frac{n ! 2}{s^{n+1}\left(e^{s}+1\right)},
$$

and also

$$
\frac{1}{e^{\lambda}+1}=\frac{1}{2}+\sum_{j=1}^{\left[\frac{1}{2}(n+1)\right]} \frac{(-1)^{j}\left(4^{j}-1\right)}{(2 j) !} B_{j} \lambda^{2 j-1}-\frac{\lambda^{n+1}}{n ! 2} \hat{H}_{n}(\lambda),
$$

these two equations being analogues of (12) and (14). Then (63) comes by taking $n=2 N-1, \lambda=\omega s$ in (65), multiplying by $\hat{x}(s)$ and interpreting the terms. Equation (63) holds for $x \in \mathbb{E}_{0}^{2 N}$.

6.3 Periodic Euler-Maclaurin summation formula of Berndt and Schoenfeld. This formula includes and generalizes the Euler-Maclaurin and the Boole formulae. It is determined by first choosing any periodic sequence $A=\left(a_{j}\right)_{j=-\infty}^{\infty}$. Let $\kappa(\in \mathbb{N})$ denote the period of $A$. Periodic Bernoulli numbers $B_{n}(A)$ and periodic Bernoulli polynomials $B_{n}(t, A)$ are defined (neither sequence nor function is actually periodic), and the formula can then in the present spirit be formulated as follows: for $x \in \mathbb{C}_{0}^{n}$,

$$
\begin{aligned}
S_{\kappa \omega} W_{A, \omega} x(t)= & \frac{B_{0}\left(A^{*}\right)}{\omega} \int_{0}^{t} x(u) d u+B_{1}\left(A^{*}\right) x(t) \\
& +\sum_{j=2}^{n} \frac{B_{j}\left(A^{*}\right)}{j !} \omega^{j-1} x^{(j-1)}(t)-\frac{\omega^{n-1}}{n !} \int_{0}^{t} V_{n}\left(\frac{t-u}{\omega}, A\right) x^{(n)}(u) d u,
\end{aligned}
$$

where the operator $W_{A, \omega}$ and function $V_{n}$ are defined below, by (77) and (80) respectively; $V_{n}$ is a modified periodic extension of $B_{n}(\cdot, A)$ outside $[0, \omega)$. We shall adopt the notation of B. C. Berndt [2], except for writing the period of $A$ as $\kappa$, not $k$, who introduces the function and sequence

$$
G(\lambda, A)=\sum_{n=0}^{\kappa-1} a_{n} e^{2 \pi i n \lambda / \kappa}, \quad A^{*}=\left(a_{-j}\right)_{j=-\infty}^{\infty} .
$$

Of more interest to us is the function

$$
\mathscr{G}(\lambda, A)=\left(e^{\kappa \lambda}-1\right)^{-1} G\left(\frac{\kappa \lambda}{2 \pi i}, A\right)=\left(e^{\kappa \lambda}-1\right)^{-1} \sum_{n=0}^{\kappa-1} a_{n} e^{n \lambda} .
$$


The definitions of $B_{j}(A)$ and $B_{j}(t, A)$ by generating functions are

$$
\begin{array}{cc}
\lambda \mathscr{G}(\lambda, A)=\sum_{j=0}^{\infty} \frac{B_{j}(A) \lambda^{j}}{j !} \quad\left(|\lambda|<\frac{2 \pi}{\kappa}\right), \\
\lambda e^{\lambda t \mathscr{G}\left(\lambda, A^{*}\right)}=\sum_{j=0}^{\infty} \frac{B_{j}(t, A) \lambda^{j}}{j !} \quad\left(|\lambda|<\frac{2 \pi}{\kappa}\right) .
\end{array}
$$

Now using the periodicity of $A$ we find that

$$
\mathscr{G}(\lambda, A)+\mathscr{G}\left(-\lambda, A^{*}\right)=-a_{0} .
$$

This gives

$$
\begin{array}{cc}
B_{j}(A)=(-1)^{j} B_{j}(0, A) \quad(j \neq 1), & B_{1}(A)=-B_{1}(0, A)-a_{0}, \\
B_{j}\left(0, A^{*}\right)=B_{j}(A) & (\text { all } j) .
\end{array}
$$

We can also deduce from (71) that

$$
B_{n}(t, A)=\sum_{j=0}^{n}\left(\begin{array}{l}
n \\
j
\end{array}\right) B_{j}(0, A) t^{n-j} .
$$

Then (72) and (73) imply for the Laplace transform of $B_{n}(\cdot, A)$ that, when $\operatorname{Re}(\lambda)>0$,

$$
\frac{\lambda^{n}}{n !} \hat{B}_{n}(\lambda, A)=\sum_{j=0}^{n} \frac{B_{j}\left(A^{*}\right)}{j !} \lambda^{j-1}
$$

is the sum of the first $n+1$ terms of the Laurent series of $\mathscr{G}\left(\lambda, A^{*}\right)$ about $\lambda=0$. Here we have an analogue of (13). Write the remainder as $-\left(\lambda^{n} / n !\right) \hat{V}_{n}(\lambda, A)$, thereby defining $V_{n}$ and giving

$$
\hat{B}_{n}(\lambda, A)=\hat{V}_{n}(\lambda, A)+\frac{n !}{\lambda^{n}} \mathscr{G}\left(\lambda, A^{*}\right),
$$

which can also be written

$$
\mathscr{G}\left(\lambda, A^{*}\right)=\sum_{j=0}^{n} \frac{B_{j}\left(A^{*}\right)}{j !} \lambda^{j-1}-\frac{\lambda^{n}}{n !} \hat{V}_{n}(\lambda, A) .
$$

These equations are the relevant analogues of (12) and (14) respectively. To reproduce the arguments of Sections 2, 3, 4 we need: (i) to identify $\mathscr{G}\left(\lambda, A^{*}\right)$ as the Laplace transform associated with some operator, and (ii) to identify $V_{n}$ as some form of periodic extension of $B_{n}(\cdot, A)$. 
As to the first, let $W_{A, \omega}$ be the operator defined by

$$
\left(W_{A, \omega} x\right)(t)=\sum_{r=1}^{\min \{\kappa,[t / \omega]\}} a_{r} x(t-r \omega) .
$$

(Thus $W_{A, \omega} x(t)=0$ for $0 \leqslant t<\omega$.) Using the periodicity of the sequence $A$ we find that

$$
\left(S_{\kappa \omega} W_{A, \omega} x\right)^{\wedge}(s)=\mathscr{G}\left(\omega s, A^{*}\right) \hat{x}(s)
$$

and also

$$
S_{\kappa \omega} W_{A, \omega}=W_{A, \omega} S_{\kappa \omega} .
$$

Here (78) is the required result for (i), and the analogue for (16): the sought operator is the product $S_{\kappa \omega} W_{A, \omega}$.

As for (ii), it turns out that

$$
V_{n}(t, A)=B_{n}\left(t-r, A^{T^{r}}\right) \quad \text { for } r \leqslant t<r+1, r=0,1, \ldots, \kappa-1,
$$

and $V_{n}(\cdot, A)$ has period $\kappa$. Here $T$ is the backward translation operator on sequences:

$$
\left(A^{T}\right)_{j}=a_{j+1} \quad \text { for all } j \in \mathbf{Z},
$$

and $A^{T^{r}}=\left(A^{T^{r-1}}\right)^{T}, A^{T^{0}}=A$ for all $r \in \mathbb{N}$.

To get (66) we write $\lambda=\omega s$ in (76), multiply by $\hat{x}(s)$ and take the inverse transforms, as we did in Section 4 . We finish by outlining the proof of $(80)$. For this, two lemmas are required.

LEMMA 11. For any sequence $A=\left(a_{j}\right)_{j=-\infty}^{\infty}$ of period $\kappa$ we have

$$
\mathscr{G}\left(\lambda, A^{T^{\prime} *}\right)=e^{r \lambda} \mathscr{G}\left(\lambda, A^{*}\right)-\sum_{p=1}^{r} a_{p} e^{(r-p) \lambda}
$$

for $r=0,1,2, \ldots$ and $\lambda \not \equiv 0(\bmod 2 \pi i / \kappa)$. Hence

$$
B_{n}\left(t, A^{T^{r}}\right)=B_{n}(t+r, A)-n \sum_{j=1}^{r} a_{j}(t+r-j)^{n-1}
$$

for $r=0,1, \ldots, n=1,2, \ldots, t \in \mathbb{R}$.

Proof. Equation (82) for the case $r=1$ follows from straightforward manipulation of the formula (68) for $\mathscr{G}\left(\lambda, A^{T *}\right)$; note that $\left(A^{T *}\right)_{j}=a_{-j+1}$, and $A^{T *} \neq$ $A^{* T}$ unless $\kappa=2$. For general $r$ the formula follows by induction.

To prove (83), we multiply (82) by $\lambda e^{\lambda t}$ and use the definition (70), getting

$$
\text { - } \sum_{n=0}^{\infty} \frac{1}{n !} B_{n}\left(t, A^{T}\right) \lambda^{n}=\sum_{n=0}^{\infty} \frac{1}{n !} B_{n}(t+1, A) \lambda^{n}-a_{1} \lambda e^{\lambda t} \text {. }
$$


Then comparison of coefficients of $\lambda^{n} / n$ ! gives (83) for the case $r=1$; and subsequent cases again follow by induction.

LEMMA 12. For $n, j=0,1, \ldots$ and $t \in \mathbb{R}$,

$$
B_{n}(t+j \kappa, A)=B_{n}(t, A)+n \sum_{p=0}^{\kappa-1} a_{-p} \sum_{l=0}^{j-1}(t+l \kappa+p)^{n-1}
$$

Proof. The case $j=1$ is Proposition 9.6 of Berndt and Schoenfeld [1]; induction gives the general case.

LEMMA 13. Equation (80) holds, where $V_{n}$ is the inverse Laplace transform of $\hat{V}_{n}$, defined by (75).

Proof. Let $U_{n}(t, A)$ denote the righthand side of (80) for $0 \leqslant t<\kappa$ and let $U_{n}$ have period $\kappa$. We have to prove (75) with $U$ written in place of $V$ (call this equation (75U)). Now

$$
\hat{U}_{n}(\lambda, A)=\frac{e^{\lambda \kappa}}{e^{\lambda \kappa}-1} \int_{0}^{\kappa} e^{-\lambda u} U_{n}(u, A) d u
$$

by periodicity, and

$$
\begin{aligned}
\int_{0}^{\kappa} e^{-\lambda u} U_{n}(u, A) d u= & \sum_{r=0}^{\kappa-1} \int_{r}^{r+1} e^{-\lambda u} B_{n}\left(u-r, A^{T^{r}}\right) d u \\
= & \sum_{r=0}^{\kappa-1} e^{-\lambda r} \int_{0}^{1} e^{-\lambda t} B_{n}\left(t, A^{T^{r}}\right) d t \\
= & \sum_{r=0}^{\kappa-1} \int_{r}^{r+1} e^{-\lambda u} B_{n}(u, A) d u \\
& -n \sum_{r=1}^{\kappa-1} \sum_{j=1}^{r} a_{j} \int_{r}^{r+1} e^{-\lambda u}(u-j)^{n-1} d u
\end{aligned}
$$

by (83); so

$$
\hat{U}_{n}(\lambda, A)=\left(1-e^{-\lambda \kappa}\right)^{-1} \int_{0}^{\kappa} e^{-\lambda u} B_{n}(u, A) d u-I_{1}(t),
$$


say. On the other hand,

(87)

$$
\begin{aligned}
\hat{B}_{n}(\lambda, A)= & \sum_{j=0}^{\infty} \int_{j \kappa}^{(j+1) \kappa} e^{-\lambda u} B_{n}(u, A) d u \\
= & \sum_{j=0}^{\infty} \int_{0}^{\kappa} e^{-\lambda(t+j \kappa)} B_{n}(t, A) d t \\
& +n \sum_{j=0}^{\infty} \sum_{p=0}^{\kappa-1} a_{-p} \sum_{l=0}^{j-1} \int_{0}^{\kappa} e^{-\lambda(t+j \kappa)}(t+l \kappa+p)^{n-1} d t \\
= & \left(1-e^{-\lambda \kappa}\right)^{-1} \int_{0}^{\kappa} e^{-\lambda t} B_{n}(t, A) d t+I_{2}(t),
\end{aligned}
$$

say. Then $\hat{B}_{n}(\lambda, A)-\hat{U}_{n}(\lambda, A)=I_{2}(t)+I_{1}(t)$. In the term $I_{2}(t)$ we reverse the order of the $j$ and $l$ summations, write $a_{\kappa-p}$ in place of $a_{-p}$ and sum over $q=\kappa-p$ in place of $p$. Similarly in $I_{1}(t)$ we reverse the order of summation; we get

$$
\begin{aligned}
I_{2}(t)+I_{1}(t)= & n\left(1-e^{-\lambda \kappa}\right)^{-1} \sum_{q=1}^{\kappa} a_{q} \int_{\kappa}^{\infty} e^{-s v}(v-q)^{n-1} d v \\
& +n\left(1-e^{-\lambda \kappa}\right)^{-1} \sum_{j=1}^{\kappa} a_{j} \int_{j}^{\kappa} e^{-\lambda u}(u-j)^{n-1} d u \\
= & n\left(1-e^{-\lambda \kappa}\right)^{-1} \sum_{j=1}^{\kappa} a_{j} \int_{j}^{\infty} e^{-\lambda u}(u-j)^{n-1} d u \\
= & -n ! \lambda^{-n}\left(\mathscr{G}(-\lambda, A)+a_{0}\right)=n ! \lambda^{-n} \mathscr{G}\left(\lambda, A^{*}\right)
\end{aligned}
$$

by $(71)$. This proves $(75 \mathrm{U})$.

The proof of the summation formula (66) proceeds as previously explained. The formula holds for all sequences $A$ of period $\kappa$, all $\omega>0, t>0, n \in \mathbb{N}$.

The function $V_{n}(t, A)$ coincides with the function $n !(-1)^{n} P_{n}\left(-t, A^{*}\right)$ of [2], equation (2.10). Our equation (66) gives (by specializing $t$ ) the starred form of the equation in [2], Theorem 3.3. The Euler-Maclaurin formula is the case $a_{j} \equiv 1$, $\kappa=1$; Boole's formula is the case $a_{j} \equiv(-1)^{j}, \kappa=2$.

\section{References}

[1] B. C. Berndt and L. Schoenfeld, 'Periodic analogues of the Euler-Maclaurin and Poisson summation formulas with applications to number theory', Acta Arith. 28 (1975), 23-68.

[2] B. C. Berndt, 'Periodic Bernoulli numbers, summation formulas and applications', (Theory and application of special functions, Academic Press, New York, 1975). 
[3] G. H. Hardy, Divergent series (Oxford University Press, 1949).

[4] E. Hille and R. S. Phillips, Functional analysis and semi-groups (Amer. Math. Soc. Colloquium Publ. 31, Providence, R. I., 1957).

[5] J. B. Miller, 'The Euler-Maclaurin sum formula for an inner derivation', Aequationes Math. 25 (1982), 42-51.

[6] J. B. Miller, 'The Euler-Maclaurin sum formula for a closed derivation', J. Austral. Math. Soc. Series A 37 (1984), 128-138.

[7] J. B. Miller, 'The Euler-Maclaurin formula generated by a summation operator', Proc. Roy. Soc. Edinburgh Sect. $A 95$ (1983), 285-300.

[8] J. B. Miller, 'Series like Taylor's series', Aequationes Math. 26 (1983), 208-220.

[9] J. B. Miller, 'The operator remainder in the Euler-Maclaurin formula', Aequationes Math. 28 (1985), 64-68.

[10] D. V. Widder, The Laplace transform (Princeton Univ. Press, 1946).

[11] W. Walter, 'Remark', Aequationes Math. 26 (1983), 281-282.

Department of Mathematics

Monash University

Clayton, Victoria 3168

Australia 